\title{
The Human Olfactory System: Cortical Brain Mapping Using fMRI
}

\author{
Faezeh Vedaei, ${ }^{1}$ Mohammad Ali Oghabian, ${ }^{2,}{ }^{*}$ Kavous Firouznia, ${ }^{1}$ Mohammad Hossein Harirchian, ${ }^{3}$ \\ Younes Lotfi, ${ }^{4}$ and Mohammad Fakhri ${ }^{2}$ \\ ${ }^{1}$ Advanced Diagnostic and Interventional Radiology Research Center, Medical Imaging Center, Imam Khomeini Hospital Complex, Tehran University of Medical Sciences, \\ Tehran, Iran \\ ${ }^{2}$ Neuroimaging and Analysis Group (NIAG), Research Center for Molecular and Cellular Imaging (RCMCI), Tehran University of Medical Sciences, Tehran, Iran \\ ${ }^{3}$ Iranian Center of Neurological Researches, Imam Khomeini Hospital, Tehran University of Medical Sciences, Tehran, Iran \\ ${ }^{4}$ Audiology Department, University of Social Welfare and Rehabilitation Sciences, Tehran, Iran \\ "Corresponding author: Mohammad Ali Oghabian, Neuroimaging and Analysis Group (NIAG), Research Center for Molecular and Cellular Imaging (RCMCI), Tehran University \\ of Medical Sciences, Tehran, Iran. Tel: +98-2166907519, Fax: +98-2166581533, E-mail: oghabian@sina.tums.ac.ir
}

Received 2013 December 14; Revised 2015 January 18; Accepted 2015 June 06.

\begin{abstract}
Background: Functional magnetic resonance imaging (fMRI) is a non-invasive and convenient method of mapping brain activity associated with the human sensory systems. Among these systems there is a lack of data from olfactory studies, which could be attributed to technical difficulties in odor delivery during scanning. The current study took advantage of an olfactometer to evaluate brain activity during the odor-smelling process.

Objectives: This study aimed to investigate the brain regions of the human olfactory system via fMRI brain imaging. A speculative survey was used to highlight the differences between studies conducted in healthy populations using olfactory tasks, as well as conducting literature survey in terms of the technical principles applying through these tasks in fMRI studies.

Subjects and Methods: A functional map of the olfactory system that used a block design, alternating between odor and non-odor phases, was examined in 15 healthy volunteers. The general linear model was used to identify statistically significant voxels that showed activation during the activation blocks.

Results: Primary and secondary olfactory regions, including the piriformis, insula, amygdala, parahippocampal gyrus, caudate nucleus, inferior frontal gyrus, middle frontal gyrus, superior temporal gyrus, and cerebellum, demonstrated significant activation in response to odor stimulation.

Conclusion: Activation of the aforementioned brain areas, and the pattern of activation, is largely in accordance with previous published olfactory studies carried out in healthy individuals.
\end{abstract}

Keywords: Cortex, Olfactory, fMRI, Healthy Human, Odor, Smell Sense

\section{Background}

Our knowledge of the field of functional brain anatomy has been drastically improved with the advent of functional magnetic resonance imaging (fMRI), a highly effective method that has allowed the mapping of numerous sensory and motor functions. However, the number of studies focusing on the functional neuroanatomy of the olfactory system has remained very small $(1,2)$. fMRI studies of the olfactory system have certain limitations that are not that important with regard to other sensory and motor tasks. Indeed, it is difficult to quantitatively explore the olfactory system, which may be due to the lack of sufficient strategies to enable stimulation of this system in a selective and controllable manner. Despite these shortcomings, the mapping of the central cortical pathways that process olfactory stimuli is of great importance because of the close connection between olfactory impairments and the early stages of some neurodegenerative disorders, such as Alzheimer's disease, Parkinson's disease, and schizophrenia. Since olfactory dysfunction is one of the earliest signs of these conditions, and this functional decline precedes the onset of motor symptoms, many studies have proposed the use of olfactory functional imaging evaluation, despite the complexity of design involved $(3,4)$. Another common clinical setting that highlights the importance of olfactory dysfunction is encountered in patients with post-traumatic hyposmia that is accompanied by frontal lobe injury, as well as temporal lobe injury $(5,6)$.

Considering the importance of conducting fMRI studies, the technical problems regarding odor delivery in the MR apparatus must be addressed. The majority of fMRI studies take advantage of block-designed tasks, which compare alternating intervals of stimulus and no-stimulus epochs with regard to increasing statistically significant changes in blood oxygen level-dependent (BOLD) signal intensity. In olfaction studies, one condition can be odor presentation (an experimental phase), while the other in- 
volves a no-odor condition (a control phase) (1, 7-9). In order to produce a precise and adequate delivery of the odor stimulus in both phases, the majority of recent studies have utilized an olfactometer device (7). To avoid any disturbance in the magnetic field, and diminishing of the signal-to-noise ratio, parts of the olfactometer that are located in the magnet room should be made from diamagnetic materials. Both the odor and no-odor (rest) phases should be executed without any thermal, tactile, and acoustic cues when switching between the two periods. Consequently, the odor and pure air should be at the same temperature and pressure. The no-odor phase involves exposure to pure air without any pollution by odor molecules. In addition, a standardized odor with a high level of replication in repetitive experiments is used for stimulation $(7,8,10)$.

To date, previous studies have demonstrated that the olfactory tract sends a signal to the central olfactory systems, including the piriformis and entorhinal cortices in the temporal lobe, as well as cortical subcortical structures, such as the amygdala. Moreover, the olfactory tract sends signals to a complex circuit that includes the thalamus, hypothalamus, dorsolateral frontal cortex, and orbitofrontal cortex (OFC). Further processing of odor characteristics and conscious analysis of odor stimuli occurs in various sections of the limbic system $(2,11)$.

\section{Objectives}

The current study aimed to explore the primary and secondary regional brain activation of olfactory areas in a healthy group of the Iranian population during an olfaction task using eucalyptus odorant. For the first time, the present study also describes the feasibility of olfactometer utilization in carrying out fMRI studies in Iran. In addition, we have described some of the methodological aspects regarding utilization of olfactory tasks in fMRI studies.

\section{Subjects and Methods}

\subsection{Participants}

A total of 15 , healthy, right-handed individuals (six men, nine women) participated. The primary investigator explained the entire study protocol to all of the participants, after which their informed consent was obtained. The study was reviewed and approved by the institutional board of Tehran University of Medical Sciences. All participants were non-smokers, and were aged between 22 and 38 years (mean $\pm S D ; 30 \pm 5$ ). A careful history was obtained from all participants, and none had nasal and sinus complaints or neurological and psychiatric diseases. To establish the healthy status of the participants with regard to possible confounding diseases, a complete neurological examination was performed by a neurologist who was unaware of the study aims.

\subsection{Olfactory Testing}

The standardized psychophysical olfactory test, the "Sniffin Stick" test, was performed with all participants before the fMRI session. This battery of tests consisted of odor threshold ( $\mathrm{T}$ ), discrimination (D), and identification (I). The total score (TDI) was determined by the sum of the three scores $(\mathrm{T}+\mathrm{D}+\mathrm{I})(12)$. For this behavioral assessment, the odorants were presented via a pen-like dispensing device. The examiner removed the pen's cap and placed it 2 centimeters away from both nostrils for approximately 3 seconds. The threshold test was conducted with phenyl ethyl alcohol or n-butanolin in a triple-forcedchoice paradigm. Three pens were presented to the participants in a randomized manner: only one of these contained the odorant, while the two others contained only the diluents. Each participant was then asked to determine the pen that smelled different from the other pens. A particular odorant concentration was only correctly identified if the pen containing the odorant was recognized twice in a row. Following this, the next highest dilution step was presented. If this was also correctly identified twice, again the next highest dilution step was presented and so on, until the participant made an incorrect decision, in which case, the next lowest dilution step was presented. If the participant could not identify this level of concentration, the next lowest dilution step was presented. This pattern was continued until the participant correctly identified a dilution step. The test was completed when seven reversal points were passed through, and the smell threshold ( $\mathrm{T}$ ) was defined as the mean of the last four staircase reversal points. For the odor discrimination test (D), 16 pen triplets were presented to each participant: two pens smelled the same, and the remaining pen contained a different odorant. Therefore, the participants had to determine which of the three pens smelled different. During the odor threshold and odor discrimination tests, the participants were blindfolded to prevent visual recognition of the pen's odorants. The odor identification test was conducted via the use of 16 common odorants. Each participant received a multiple-choice card and was asked to pick the term that described the presented odorant. For all three parts of the test (threshold, discrimination, and identification), the participants' scores ranged from 1 to 16 . Therefore, a total score was determined by totaling the results obtained (TDI score). Accordingly, olfactory function could be classified in terms of anosmia (TDI < 16), hyposmia $(16<$ 
TDI < 30), and normosmia (TDI > 30) $(7,12)$. The results of this test ensured that all of the participants had a normal olfactory system. A score of $\geq 30$ was utilized to identify participants that were appropriate for the fMRI session.

\subsection{FMRI Data Acquisition}

Functional MRI was performed to measure BOLD contrasts on a 3-Tesla (Siemens, MAGNETOM Trio; Germany) MR-system. During the scanning procedure, foam cushions were used to minimize movements of the head within the coil. Each imaging session began with acquisition of a high-resolution, T1-weighted, three-dimensional anatomical scan. A T1-weighted spin-echo sequence was used to generate high-resolution structural maps of the participants' brains, with the same dimension and orientation as the fMRI ( $\mathrm{TR}=1800 \mathrm{~ms}$; $\mathrm{TE}=90 \mathrm{~ms}$; flip angle $=90^{\circ}$ ). The fMRI data were obtained using a gradient-echo echoplanar images (EPIs) protocol (TE = $60.3 \mathrm{~ms}$; TR = $3125 \mathrm{~ms}$; flip angle $=90^{\circ}$; field of view $=22 \mathrm{~cm}^{2}$; number of slices $=$ 15; slice thickness $=6 \mathrm{~mm}$; spacing $=0 \mathrm{~mm}$; bandwidth $=$ $5.62 \mathrm{kHz}$ ). Total fMRI acquisition lasted 10 minutes for each participant.

\subsection{FMRI Activation Task}

Olfactory stimulation was administered using a Magconcept olfactometer (USA, 2010) with a continuous airflow rate ( $2 \mathrm{~L} / \mathrm{minute}$ ). The olfactometer device consisted of two main parts; a positive airway pressure system and an odorant delivery system. This system was controlled by a computer software program to permit choosing of, and switching between, different types of stimulation. Odor and air delivery timings, stimulus frequency, and choice of a specific odorant for stimulus presentation within any task were controlled by the software.

The odorant presentation model that we used was a block design that consisted of two alternating patterns. A 15-second odor presentation (eucalyptus; nature's alchemy Co. $100 \%$ pure natural essential oil), followed by a rest period of 45 seconds (odorless air), constituted these blocks. This alternation of activation and rest was repeated for 10 rounds (a total of 600 seconds).

\subsection{Technical Aspects}

Several technical considerations should be applied in every brain olfactory fMRI study, some of which are related to the participants. They should be asked to breath normally without sniffing during the scanning, and to not move their head at all $(7,13-16)$.

In addition to the technical aspects relating to participants that should be considered during olfactory fMRI studies, several specific methods should be used to precisely deliver odor molecules to the scanner. A method used in the past was to place a piece of odor-saturated cotton in front of the participant's nose. This method is the most helpful in positron emission tomography(PET) imaging studies, but it is not reliable in fMRI studies due to the possibility of tactile stimuli during the scanning if the cotton touches the participant's nose. Moreover, concentration level of the odorant cannot be controlled, so the odor stimuli would not be precise. Nowadays, a more advanced method is used: a mobile olfactometer device to produce and convey the odorant stimuli to the participant's nose during scanning, which does not have the limitations of the previously described methods. These tools consist of three main parts: a positive air pressure device that is used in the control room, a nasal mask, and a part that consists of some capsules that contain the odorants. This unit was used in the magnet room and is controlled by computerbased software that provides the facility to switch between the rest and odor stimulus phases. In addition, it allows the programmer to maintain desirable timing and frequency of the olfactory stimuli task.

Some technical matters must be addressed in designing olfactometer devices and performing olfactory tasks. For example, some parts of the olfactometer that are placed in the magnet room should be made by diamagnetic materials to avoid any uniformity disturbances of the magnetic field and a decline of signal-to-noise ratio. In order to create a desirable olfactory task during scanning, olfactometers should have the capacity to deliver a variety of odorants over a random and selectable duration. The computer-based software in this system supports this requirement. As most of the olfactory tasks consist of odorless phases, this state should not be polluted by any odorant molecules of the stimulus phases and should contain pure air. Furthermore, to prevent tactile and thermal stimuli during scanning in both rest and stimulus phases, the pure air and odorant that is conveyed to the participant's nose should be at the same pressure and temperature ( 8 , 17-19).

\subsection{Data Analysis}

Image pre-processing and statistical analysis were conducted using functional magnetic resonance imaging of the brain (FMRIB) software library (FSL) software package (FMRIB's software library, http://www.fmrib.ox.ac.uk/fsl) version 5.2.0 and the fMRI expert analysis tool (FEAT). The pre-processing steps were executed on EpiData software and consisted of brain extraction to discharge signals that were not related to brain tissue signals using the brian extraction tool (BET, v. 2. 1; FSL), slice-timing correction applying phase-shifting in Fourier time-series space, motion 
correction by the FMRIB's linear image registration tool (MCFLIRT; FSL), application of spatial smoothing with a 5$\mathrm{mm}$ full width half maximum isotropic Gaussian kernel, and non-linear, high-pass temporal filtering, with a cut-off frequency of 60 seconds. Moreover, 4D data normalization to the standard stereotactic space (Talairach and Tournoux, 1988) was performed. The parametric statistical analysis was then carried out using FEAT analysis, which is based on the general linear model. In FEAT, the first-level analysis (time series for single-participant analysis), known as the FMRIB Improved linear model, utilizes the precise nonparametric estimation of a time series to generate the optimum estimation efficiency. The corresponding BOLD signal was characterized by a "z-score" that was achieved by means of a t-test and dividing the parameter estimate by its standard error. At the final stage, cluster thresholding was performed to clarify those clusters with statistically significant activations. Hence, only clusters with a z-statistic greater than 2.3 and a P value of cluster threshold of less than 0.05 were regarded as statistically significant activations.

For group analysis, higher-level analysis FEAT used FMRIB's local analysis of mixed effects to model and estimate the random-effects component of the measured variances of the inter-session mixed-effects. All activation maps were overlaid and registered onto a standard Montreal neurological institute brain template implanted in FSL software.

\section{Results}

\subsection{Behavioral Results}

Behavioral olfactory testing was carried out before the fMRI session, in order to ensure the intact olfactory status of the participants and to exclude hyposmic and anosmic cases. We specified that participants have a TDI score of $\geq$ 30 , and our results showed that the mean TDI score of the "Sniffin Stick" test was $33(\mathrm{SD}=3.2$; range $=30-36)$. As we had expected, healthy participants were identified via an average TDI score of more than 30 , while three volunteers with a TDI score of less than 30 were excluded from our study.

\subsection{Imaging Data}

Figure 1, a multi-subject, higher-level, random effect analysis across 15 healthy individuals, shows a brain activation map while eucalyptus is being smelled. As is evident in Table 1, significant activation was observed in bilateral primary olfactory structures, including the piriform cortex (Brodmann area 34), insula, and amygdala, as well as secondary olfactory regions, such as the parahippocampal gyrus, caudate, middle and superior frontal gyrus, OFC, and superior temporal gyrus. In addition, activation of the right cerebellum was observed in the activation map.

\section{Discussion}

This study aimed to identify the cerebral regions that are involved in the smelling of an odorant. To achieve this aim, we stimulated the olfactory system with a pleasant odorant, while fMRI scanning was performed. The activation we found was in accordance with the results of previous fMRI and PET findings. We determined odorinduced neural responses in some olfactory areas, including the piriform cortex, amygdala, insula, parahippocampal gyrus, inferior frontal gyrus, middle frontal gyrus, and cerebellum. Most of the observed regions have been described in previous literature, and are separately discussed in the following paragraphs.

\subsection{Piriform Cortex}

Olfactory-evoked activation of the piriform cortex has been inconsistently identified across previously fMRI and PET studies $(1,20)$. Zatorre et al. (1992) were among the first to determine odor-evoked activation in the pirifrom cortex in humans (21). Other PET and fMRI studies have shown that odors have the capacity to induce activity in this area, but the activity observed has been inconsistent. Moreover, some studies have revealed only insignificant activity in this region during olfactory stimulation. In addition, several studies have failed to exhibit piriform cortex activity during the smelling of an odorant. This finding is surprising, as a comparison of the primary olfactory cortex (POC) with the robust and consistent activation in other primary sensory systems revealed that POC activation is not as prominent as that found in other primary sensory cortices. Conversely, the secondary olfactory cortex is capable of showing a high level of activation during olfactory tasks $(20,22-24)$. To address this difference, the timing of responses should be considered, since habituation plays an important role in reduction of neural activity in the POC. Some studies have shown that the piriform cortex and other parts of the primary olfactory cortex are activated at the beginning of exposure to olfactory stimuli (the first 10 - 15 seconds), after which the activation signal returns to its baseline level (20). Hence, habituation is the most important explanation for the inconsistencies in piriform activity. In addition, a magnetic susceptibility artifact may reduce the signal-to-noise ratio in this region $(1,20,22$, 25).

It should be noted that smelling and sniffing are two distinct functions, both of which are engaged during a typical olfactory stimulation $(23,26)$. Sniffing and nasal airflow can induce piriform cortex activity in the presence 


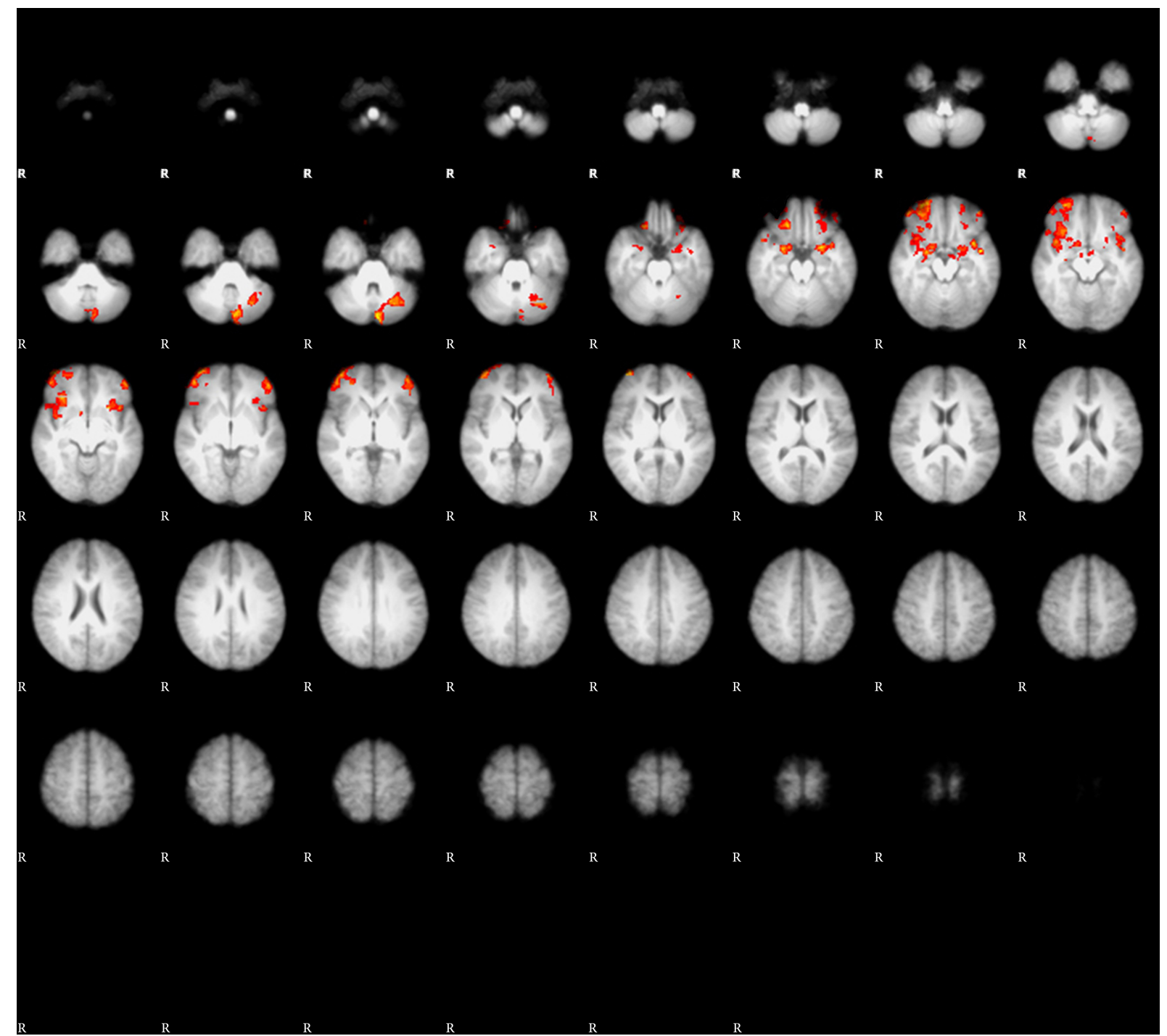

Figure 1. Regional brain activation during the olfactory stimulation task. Axial sections of functional magnetic resonance imaging group analysis were registered to the T1 standard space image with functional magnetic resonance imaging of the brain (FMRIB) software library with an analysis threshold of z-stat $>2.3(P<0.05)$.

and absence of odorant stimuli: Kareken et al. (2004) showed piriform cortex activity as a result of odorless sniffing. Indeed, the sensation of nasal airflow is sufficient to induce piriform cortex activity in all scans, but it is subtracted in data analysis of block designs (26). In the present study, we found that the right piriform cortex had a higher Z-score than its left counterpart. This is in agreement with previous reports that studied the piriform cortex in olfactory tasks; Plailly et al. also reported greater activation in the right piriform cortex than in the left piriform cortex. This finding might be attributed to the role of the right piriform cortex in familiarity judgment tasks (25).

\subsection{Hippocampus/Insula}

Considerable research has shown that not only does the primary olfactory cortex demonstrate habituation during olfactory stimulation, but the hippocampus and anterior insula are also expected to show the same time-course in their response (20). Indeed, these areas have a close functional interaction during an extended olfactory stimulation. The hippocampus is anatomically linked to the primary olfactory cortex (piriform via the entorhinal cortex) (27). Moreover, the anterior insula receives olfactory projections directly from the piriform and other areas of the POC, such as the amygdala $(28,29)$. All of these areas may 
Table 1. Brain Regions Activated Under Odorant Stimulation that Were Found with a Group Analysis of All Participants (The Coordinates are From a Multi-Subject Analysis)

\begin{tabular}{|c|c|c|c|c|c|}
\hline \multirow{2}{*}{$\begin{array}{l}\text { Brain Region } \\
\text { Local Maxima Regions }\end{array}$} & \multicolumn{3}{|c|}{ Talairach Coordinate } & \multirow{2}{*}{ Voxels } & \multirow{2}{*}{ Z-stat } \\
\hline & $\mathbf{X}$ & $\mathbf{Y}$ & $\mathbf{Z}$ & & \\
\hline $\begin{array}{l}\text { Left cerebrum, limbic lobe, } \\
\text { parahippocampal gyrus }\end{array}$ & -24 & 4 & -20 & 118 & 4.3 \\
\hline $\begin{array}{l}\text { Left cerebrum, temporal lobe, } \\
\text { superior temporal gyrus }\end{array}$ & -44 & -2 & -18 & 915 & 4.3 \\
\hline Left cerebellum, posterior lobe, uvula & -6 & -86 & -32 & 544 & 4.3 \\
\hline $\begin{array}{l}\text { Right cerebrum, frontal lobe, } \\
\text { superior frontal gyrus }\end{array}$ & 30 & 56 & -14 & 2600 & 4.2 \\
\hline Left insula & -26 & 16 & -6 & 370 & 3.8 \\
\hline Right orbital frontal cortex & 18 & 30 & -20 & 115 & 3.8 \\
\hline Right amygdala & 24 & -2 & -16 & 105 & 3.8 \\
\hline $\begin{array}{l}\text { Left cerebrum, temporal lobe, inferior } \\
\text { temporal gyrus }\end{array}$ & -36 & 8 & -16 & 175 & 3.7 \\
\hline $\begin{array}{l}\text { Left cerebrum, frontal lobe, middle } \\
\text { frontal gyrus }\end{array}$ & -44 & 50 & -14 & 210 & 3.7 \\
\hline $\begin{array}{l}\text { Right cerebrum, frontal lobe, middle } \\
\text { frontal gyrus }\end{array}$ & 44 & 62 & -10 & 266 & 3.6 \\
\hline Right caudate & 12 & 12 & -10 & 65 & 3.3 \\
\hline Left amygdala & -26 & 0 & -20 & 198 & 3.2 \\
\hline $\begin{array}{l}\text { Right cerebrum, limbic lobe, } \\
\text { parahippocampal gyrus }\end{array}$ & 24 & 0 & -18 & 88 & 3.2 \\
\hline $\begin{array}{l}\text { Right piriform cortex, Brodmann area } \\
34\end{array}$ & 16 & 4 & -18 & 99 & 3.2 \\
\hline
\end{tabular}

${ }^{\mathrm{a}} \mathrm{X}, \mathrm{Y}$, and $\mathrm{Z}$ coordinates are described according to Talairach space.

${ }^{\mathrm{b}} \mathrm{P}<0.05$.

play an important role in discrimination of odor quality during olfactory tasks $(20,30)$.

\subsection{Amygdala}

Among all of the sensory systems in humans, the olfactory system has the most direct connection with the amygdala (1). Many studies have verified the activation of the amygdala during olfactory stimulation as a part of the POC. This activation is induced by both the recognition of the odorant and the emotional reaction to the odor (1). In addition, Anderson et al. found that the level of amygdala activation was in accordance with the odor intensity (31).

\subsection{Orbitofrontal Cortex}

The orbitofrontal cortex (OFC) contours the ventral surface of the frontal lobe and is a secondary cortical affiliation in the olfactory brain regions (29). It receives olfactory projections from the piriform cortex, amygdala, and mediodorsal nucleus of the thalamus. Despite the transient activation in the POC, the OFC and other parts of the secondary olfactory cortex have shown a prolonged activation during an entire stimulus presentation (29). To date, OFC activation has been perceived during olfactory discrimination and identification tasks. Some functional imaging studies have shown that the right OFC can be employed by familiarity judgment of an odor (25). Royet et al. reported that the right OFC can be activated by familiarity judgment of odor, whereas hedonic judgment of odor induces left OFC activation $(1,32)$. Indeed, analyses of people with OFC lesions have revealed that right OFC lesions may cause greater impairments in the olfactory system than left OFC lesions $(33,34)$.

The laterality of observed responses in the OFC in olfactory processing has been addressed in previous studies (25). Hummel et al. (2010) investigated the brain's response to pleasant and unpleasant odors in healthy individuals and people with Parkinson's disease. They found that left OFC activity was stronger during smelling of the hedonic odors (3). Moreover, Royet et al. showed strong lateralization of familiarity judgment of hedonic odor on 
the left of the OFC and the frontal gyrus (35). Furthermore, some parts of the OFC that lie in the posterior and medial parts of the inferior frontal lobe can be induced by sniffing, in a similar manner to the piriform cortex (25).

\subsection{Caudate}

The caudate is located in the basal ganglia and is connected to the dorsolateral circuit and the OFC. In the present study, we observed that the right caudate was significantly active during the task presentation, which is in accordance with previous findings. For example, Savic et al. explained that the right caudate could be engaged in discrimination of odor quality (30). Indeed, it is believed that this part of the basal ganglia is involved in non-motor facets of behavior, such as mood and cognition. Poellinger et al. conducted an olfactory study using both short and prolonged olfactory stimuli, and concluded that the activation of the caudate shows a short increase in signal activity that lasts for 15 - 30 seconds before decreasing to the baseline value (20).

\subsection{Cerebellum}

Several neuroimaging studies of olfaction have found odorant-induced activation of the cerebellum. Increasing evidence shows that the cerebellum is a major player in higher cognitive processes, such as odor recognition, discrimination of odor intensity, and discrimination of odor quality $(2,30,36)$. Lombion et al. observed cerebellar activation during odor stimulation with a unimodal olfactory stimulus in a study of healthy individuals (15). Activation of the cerebellum has also been reported in many previous olfactory studies, and its activation has been explained as a result of its role in emotional processing $(30,37,38)$.

\subsection{Conclusions}

The present study demonstrates that the olfactory brain areas are employed during the smelling of a hedonic and a familiar odor (Eucalyptus). We found that both primary and secondary olfactory cortices were involved. Pleasantness of the odor, timing of the task, familiarity of the presented odor, considerations regarding purity of the odorant (unimodal odorant) versus trigeminal olfactoryevoked odor (bimodal odorant) determine the activation of particular parts of established olfactory regions. Since these considerations are all important when conducting an olfactory fMRI task, certainty regarding the parameters of the presented stimuli is vital. The present study proposes the olfactometer as the optimal method of presentation. The methods indicated above can be used in addressing the activity of brain regions in olfactory processing in a variety of conditions in healthy individuals.

\section{Acknowledgments}

This work has been partially supported by the Iranian legal medicine organization. All the authors are grateful to this organization for its help.

\section{Footnotes}

Authors' Contribution: All authors participated equally in this study. Faezeh Vedaei conducted the imaging of the participants. Mohammad Ali Oghabian provided the MRI scanner for imaging. Kavous Firouznia provided interpretations of the findings. Mohammad Hossein Harirchian provided participants. Younes Lotfid helped in defining the hypotheses of the project. Mohammad Fakhri conducted data analysis.

Financial Disclosure: The authors have no financial interests related to the present manuscript.

Funding/Support: This work was financially supported by the Iranian legal medicine organization, Iran.

\section{References}

1. Zald DH, Pardo JV. Functional neuroimaging of the olfactory system in humans. Int J Psychophysiol. 2000;36(2):165-81. [PubMed: 10742571].

2. Savic I. Processing of odorous signals in humans. Brain Res Bull. 2001;54(3):307-12. [PubMed: 11287135].

3. Hummel T, Fliessbach K, Abele M, Okulla T, Reden J, Reichmann $\mathrm{H}$, et al. Olfactory FMRI in patients with Parkinson's disease. Front Integr Neurosci. 2010;4:125. doi: 10.3389/fnint.2010.00125. [PubMed: 21120143].

4. Wang J, Eslinger PJ, Doty RL, Zimmerman EK, Grunfeld R, Sun X, et al. Olfactory deficit detected by fMRI in early Alzheimer's disease. Brain Res. 2010;1357:184-94. doi: 10.1016/j.brainres.2010.08.018. [PubMed: 20709038].

5. Yousem DM, Geckle RJ, Bilker WB, Kroger H, Doty RL. Posttraumatic smell loss: relationship of psychophysical tests and volumes of the olfactory bulbs and tracts and the temporal lobes. Acad Radiol. 1999;6(5):264-72. [PubMed:10228615].

6. Doty RL, Yousem DM, Pham LT, Kreshak AA, Geckle R, Lee WW. Olfactory dysfunction in patients with head trauma. Arch Neurol. 1997;54(9):1131-40. [PubMed: 9311357].

7. Vedaei F, Fakhri M, Harirchian MH, Firouznia K, Lotfi Y, Ali Oghabian M. Methodological considerations in conducting an olfactory fMRI study. Behav Neurol. 2013;27(3):267-76. doi: 10.3233/BEN-120320. [PubMed: 23619085].

8. Sobel N, Prabhakaran V, Desmond JE, Glover GH, Sullivan EV, Gabrieli JD. A method for functional magnetic resonance imaging of olfaction. J Neurosci Methods. 1997;78(1-2):115-23. [PubMed: 9497007].

9. Borromeo S, Hernandez-Tamames J, Luna G, Machado F, Malpica N, Toledano A, et al, editors. Objetive assessment of olfactory function using functional magnetic resonance. Medical Measurements and Applications. 2009; IEEE.

10. Popp R, Sommer M, Muller J, Hajak G. Olfactometry in fMRI studies: odor presentation using nasal continuous positive airway pressure. Acta Neurobiol Exp (Wars). 2004;64(2):171-6. [PubMed:15366250].

11. Neuroscience. 3th ed. Signature associate: JSTOR; 2006. pp. 86-7. 
12. Hummel T, Sekinger B, Wolf SR, Pauli E, Kobal G. 'Sniffin' sticks': olfactory performance assessed by the combined testing of odor identification, odor discrimination and olfactory threshold. Chem Senses. 1997;22(1):39-52. [PubMed: 9056084].

13. Rolls ET, Kringelbach ML, de Araujo IE. Different representations of pleasant and unpleasant odours in the human brain. Eur J Neurosci. 2003;18(3):695-703. [PubMed: 12911766].

14. Schneider F, Habel U, Reske M, Toni I, Falkai P, Shah NJ. Neural substrates of olfactory processing in schizophrenia patients and their healthy relatives. Psychiatry Res. 2007;155(2):103-12. doi: 10.1016/j.pscychresns.2006.12.004. [PubMed: 17532193].

15. Lombion S, Comte A, Tatu L, Brand G, Moulin T, Millot JL. Patterns of cerebral activation during olfactory and trigeminal stimulations. Hum Brain Mapp. 2009;30(3):821-8. doi: 10.1002/hbm.20548. [PubMed: 18330871].

16. Vigouroux M, Bertrand B, Farget V, Plailly J, Royet JP. A stimulation method using odors suitable for PET and fMRI studies with recording of physiological and behavioral signals. J Neurosci Methods. 2005;142(1):35-44. doi: 10.1016/j.jneumeth.2004.07.010. [PubMed: 15652615].

17. Lorig TS, Elmes DG, Zald DH, Pardo JV. A computer-controlled olfactometer for fMRI and electrophysiological studies of olfaction. Behav Res Methods Instrum Comput. 1999;31(2):370-5. [PubMed:10495824].

18. Keating S. Design and Testing of an Olfactory Stimulus Presentation Device for use in Functional Magnetic Resonance Imaging (fMRI) 2010. Available from: http://hdl.handle.net/11375/14427.

19. Sommer JU, Maboshe W, Griebe M, Heiser C, Hormann K, Stuck BA, et al. A mobile olfactometer for fMRI-studies. J Neurosci Methods. 2012;209(1):189-94. doi: 10.1016/j.jneumeth.2012.05.026. [PubMed: 22683953].

20. Poellinger A, Thomas R, Lio P, Lee A, Makris N, Rosen BR, et al. Activation and habituation in olfaction-an fMRI study. Neuroimage. 2001;13(4):547-60. doi: 10.1006/nimg.2000.0713. [PubMed: 11305885].

21. Zatorre RJ, Jones-Gotman M, Evans AC, Meyer E. Functional localization and lateralization of human olfactory cortex. Nature. 1992;360(6402):339-40. doi: 10.1038/360339a0. [PubMed:1448149].

22. Zald DH, Pardo JV. Emotion, olfaction, and the human amygdala: amygdala activation during aversive olfactory stimulation. Proc Natl Acad Sci U S A. 1997;94(8):4119-24. [PubMed: 9108115].

23. Sobel N, Prabhakaran V, Desmond JE, Glover GH, Goode RL, Sullivan $\mathrm{EV}$, et al. Sniffing and smelling: separate subsystems in the human olfactory cortex. Nature. 1998;392(6673):282-6. doi: 10.1038/32654. [PubMed: 9521322].

24. Sobel N, Prabhakaran V, Zhao Z, Desmond JE, Glover GH, Sullivan $\mathrm{EV}$, et al. Time course of odorant-induced activation in the human primary olfactory cortex. J Neurophysiol. 2000;83(1):537-51. [PubMed:
10634894].

25. Plailly J, Bensafi M, Pachot-Clouard M, Delon-Martin C, Kareken $\mathrm{DA}$, Rouby $\mathrm{C}$, et al. Involvement of right piriform cortex in olfactory familiarity judgments. Neuroimage. 2005;24(4):1032-41. doi: 10.1016/j.neuroimage.2004.10.028. [PubMed: 15670680].

26. Kareken DA, Sabri M, Radnovich AJ, Claus E, Foresman B, Hector $D$, et al. Olfactory system activation from sniffing: effects in piriform and orbitofrontal cortex. Neuroimage. 2004;22(1):456-65. doi: 10.1016/j.neuroimage.2004.01.008. [PubMed: 15110039].

27. Shipley MT, Ennis M. Functional organization of olfactory system. J Neurobiol. 1996;30(1):123-76. doi: 10.1002/(SICI)10974695(199605)30:1<123::AID-NEU11>3.0.CO;2-N. [PubMed: 8727988].

28. Behan M, Haberly LB. Intrinsic and efferent connections of the endopiriform nucleus in rat. J Comp Neurol. 1999;408(4):532-48. [PubMed: 10340503].

29. Carmichael ST, Clugnet MC, Price JL. Central olfactory connections in the macaque monkey. J Comp Neurol. 1994;346(3):403-34. doi: 10.1002/cne.903460306. [PubMed: 7527806].

30. Savic I. Imaging of brain activation by odorants in humans. Curr Opin Neurobiol. 2002;12(4):455-61. [PubMed: 12139995].

31. Anderson AK, Christoff K, Stappen I, Panitz D, Ghahremani DG, Glover G, et al. Dissociated neural representations of intensity and valence in human olfaction. Nat Neurosci. 2003;6(2):196-202. doi: 10.1038/nn1001. [PubMed: 12536208].

32. Royet JP, Koenig O, Gregoire MC, Cinotti L, Lavenne F, Le Bars D, et al. Functional anatomy of perceptual and semantic processing for odors. J Cogn Neurosci. 1999;11(1):94-109. [PubMed: 9950717].

33. Jones-Gotman M, Zatorre RJ. Odor recognition memory in humans: role of right temporal and orbitofrontal regions. Brain Cogn. 1993;22(2):182-98. [PubMed: 8373572].

34. Zatorre RJ, Jones-Gotman M. Human olfactory discrimination after unilateral frontal or temporal lobectomy. Brain. 1991;114 ( Pt 1A):7184. [PubMed: 1998891].

35. Royet JP, Zald D, Versace R, Costes N, Lavenne F, Koenig O, et al. Emotional responses to pleasant and unpleasant olfactory, visual, and auditory stimuli: a positron emission tomography study. J Neurosci. 2000;20(20):7752-9. [PubMed: 11027238].

36. Ferdon S, Murphy C. The cerebellum and olfaction in the aging brain: a functional magnetic resonance imaging study. Neuroimage. 2003;20(1):12-21. [PubMed: 14527566].

37. Herz RS, Eliassen J, Beland S, Souza T. Neuroimaging evidence for the emotional potency of odor-evoked memory. Neuropsychologia. 2004;42(3):371-8. [PubMed: 14670575].

38. Qureshy A, Kawashima R, Imran MB, Sugiura M, Goto R, Okada K, et al Functional mapping of human brain in olfactory processing: a PET study. J Neurophysiol. 2000;84(3):1656-66. [PubMed:10980035]. 